\title{
Density Perturbations in the Brans-Dicke Theory
}

\author{
J. P. Baptista ${ }^{1}$, J. C. Fabris ${ }^{2}$ and S. V. B. Gonçalves ${ }^{3}$ \\ Departamento de Física \\ Universidade Federal do Espírito Santo \\ Goiabeiras - CEP 29060 -900 \\ Vitória - Espírito Santo \\ Brazil
}

July 24, 2018

\footnotetext{
${ }^{1}$ E-MAIL: PLINIO@CCE.UfES.BR

${ }^{2}$ E-MAIL: FABRIS@CCE.UFES.BR

${ }^{3}$ E-MAIL: SERGIO@CCE.UFES.BR
} 


\begin{abstract}
We give here the calculation of density perturbations in a gravitation theory with a scalar field non-minimally coupled to gravity, i.e., the Brans-Dicke Theory of gravitation. The purpose is to show the influence of this scalar field on the dynamic behaviour of density perturbations along the eras where the equation of state for the matter can be put under the form $p=\alpha \rho$, where $\alpha$ is a constant. We analyse the asymptotic behaviour of this perturbations for the cases $\alpha=0, \alpha=-1, \alpha=1 / 3$ and $\rho=0$. In general, we obtain a decaying and growing modes. In the very important case of inflation, $\alpha=-1$, there is no density perturbation, as it is well known. In the vacuum phase the perturbations on the scalar field and the gravitational field present growing modes at the beginning of the expansion and decaying modes at the end of this phase. In the case $\alpha=0$ it is possible, for some negative values of $\omega$, to have an amplification of the perturbations with a superluminal expansion of the scale factor. We can also obtain strong growing modes for the density contrast for the case where there is a contraction phase which can have physical interest in some primordial era.
\end{abstract}




\section{Introduction}

The existence of a classical scalar field in Nature has been considered in many theories of gravitation that present alternatives to General Relativity. The prototype of scalar theories is the Brans-Dicke Theory [1], [2], [3], [4], whose Lagrangian is given by:

$$
S=\frac{1}{16 \pi} \int d^{4} x \sqrt{-g}\left[\phi R-\omega\left(\frac{\phi,{ }_{\mu} \phi,^{\mu}}{\phi}\right)+16 \pi \mathcal{L}_{m a t}\right] .
$$

It can be expected that the presence of the scalar field leads to different predictions with respect to those we obtain in General Relativity. In cosmology, this can lead to far reaching consequences, since the standard scenario given by General Relativity presents, besides some spectacular success, important drawbacks such as the horizon, flatness and structure formation problems [5].

However, local physics limits the value of the parameter $\omega$ : it must be greater than 500 to account the classical tests. This result has reduced the interest in the Brans-Dicke Theory. This situation has changed recently with the proposal of the extended inflation [6],[7]; in the de Sitter phase, Brans-Dicke Theory predicts power-law inflation instead of exponential; in

fact, following D. La and P. Steinhardt, at the beginning of inflation the BD solutions (for $p=-\rho$ ) approaches the Einstein-de Sitter solution. In the second stage of the inflation, both the scalar field and the scale factor grow by power law rather than exponential. This feature prevents the so-called 
"graceful exit" problem. However, in order to work, the parameter must be $\omega \approx 24$, contradicting observation; nevertheless this constraint follows from local conditions. This drawback can be overcome through a generalization of the original Brans-Dicke Theory, allowing the parameter $\omega$ to be a function of the field $\phi$ itself.

This revival of Brans-Dicke Theory leads us to ask if the problem of structure formation can be modified through the introduction of scalar field. This question has been treated in many different situations in the literature [8], [9], [10]. Here, we propose to study the evolution of density fluctuation in the traditional Brans-Dicke Theory in the different phases of the Universe. Even if we consider $\omega$ as a constant, this analyses can furnish many insights in how the scalar field modifies the main conclusions about gravitational instability obtained employing General Relativity. In particular we will see that in de Sitter phase, the scenario differs substantially from the traditional one. In the other phases, however, the differences are much less important. As it was mentioned above, the main interest in the presence of the scalar field is the possibility that this field accelerates the growth of density perturbations.

We will work in the Lifschitz-Khalatnikov formalism [11]. The reason is that it furnishes essentially the same relevant results we can obtain employing another formalism, and also it allows for the choice of synchronous frames where the physical interpretation of the concerned quantities are easily done. We must, of course, be careful about the presence of unphysical modes [12]. However they can be eliminated by performing an infinitesimal coordinate transformation. Taking care of the so called residual coordinate freedom, we can be sure to retain just the physical modes. 
We will allow for negative values for the parameter $\omega$. In fact, a negative $\omega$ is what is predicted by the effective models comming from Kaluza-Klein and Superstring theories 13.

This paper is organized as follows. In section II we present the background solutions of the unperturbed Universe in the following phases of its development: vacuum, inflation, radiation and incoherent matter. In section III we obtain the perturbed equations and their solutions in terms of Bessel functions for that phases. In section IV we calculate the asymptotic behaviour of the solutions for $t \rightarrow 0$ and $t \rightarrow \infty$. Finally, in section $\mathrm{V}$ we discuss the relations between the wavelength $\lambda$ and the particle-horizon distance $H^{-1}$.

We will assume in this article the following notations: the greek indices run from zero to three; the latin indices run from one to three; the signature is $(+,-,-,-)$; we use a Robertson-Walker metric with flat space section $(k=0)$; the scalar field $\phi$ is a time function; the energy-momentum is the perfect fluid; we use the synchronous gauge which fix the reference frame.

\section{Background Solutions}

We assume that background Universe is spatially flat, homogeneous and isotropic, i. e., it is described by the Robertson-Walker metric:

$$
d s^{2}=d t^{2}-a^{2} d x^{2},
$$


where the $a(t)$ is a scale factor of the Universe, and $c=1$. The energymomentum tensor of the background matter takes a perfect fluid form:

$$
T^{\mu \nu}=(\rho+p) U^{\mu} U^{\nu}-p g^{\mu \nu}
$$

with an equation of state $p=\alpha \rho$. In these expressions $\rho$ is a density matter, $p$ is a pressure and $\alpha$ is a constant.

From the Lagrangian density (11), we obtain the field equations:

$$
\begin{aligned}
& R_{\mu \nu}-\frac{1}{2} g_{\mu \nu} R= \frac{8 \pi}{\phi} T_{\mu \nu}+\frac{\omega}{\phi^{2}}\left(\phi ;_{\mu} \phi ;_{\nu}-\frac{1}{2} g_{\mu \nu} \phi ;_{\rho} \phi ;^{\rho}\right)+ \\
&+ \frac{1}{\phi}\left(\phi ;_{\mu} ;_{\nu}-g_{\mu \nu} \square \phi\right), \\
& \square \phi=\frac{8 \pi}{3+2 \omega} T .
\end{aligned}
$$

Inserting the metric (2), into equations (4) and (5) we obtain the equations of motion:

$$
\begin{gathered}
-3 \frac{\ddot{a}}{a}=\frac{8 \pi}{\phi} \rho\left(\frac{2+\omega+3 \alpha+3 \alpha \omega}{3+2 \omega}\right)+\frac{\omega}{\phi^{2}} \dot{\phi}^{2}+\frac{1}{\phi} \ddot{\phi}, \\
\ddot{\phi}+3 \frac{\dot{a}}{a} \dot{\phi}=\frac{8 \pi}{3+2 \omega} \rho(1-3 \alpha) .
\end{gathered}
$$

The above equations must be supplemented by a conservation equation:

$$
T^{\mu \nu}{ }_{\nu}=0 ;
$$

when $\mu=0$ we have the energy conservation:

$$
\dot{\rho}+3 \frac{\dot{a}}{a} \rho(1+\alpha)=0
$$

while, for $\mu=j$ we get the condition:

$$
\rho, i=0
$$


Background solutions can be obtained assuming that the scale factor $a(t)$ and the scalar field $\phi(t)$ have a power-law form:

$$
a(t) \propto t^{r} \quad, \quad \phi(t) \propto t^{s} .
$$

By direct substitution of the above relation in the equations (6), (7), (9) and (10), we have:

$$
\begin{gathered}
-3 r(r-1)=8 \pi \rho t^{2-s}\left(\frac{2+\omega(1+3 \alpha)+3 \alpha}{3+2 \omega}\right)+s^{2}(1+\omega)-s, \\
s^{2}+s(3 r-1)=\frac{8 \pi}{3+2 \omega} \rho t^{2-s}(1-3 \alpha), \\
\dot{\rho}+3 \frac{r}{t} \rho(1+\alpha)=0, \\
\rho, i=0 .
\end{gathered}
$$

In what follows we shall consider the important special cases: vacuum, inflation, radiation and incoherent matter.

\section{$2.1 \quad$ Vacuum $\quad(\rho=0)$}

Here, the Universe has no ordinary matter and the energy-momentum tensor is null in all space-time. Our background solutions from equations (12), (13) are

$$
s=1-3 r \quad, \quad r=\frac{\omega+1 \pm \sqrt{1+\frac{2 \omega}{3}}}{4+3 \omega}
$$

where $\omega>-\frac{3}{2}$. 


\subsection{Inflation $(\alpha=-1)$}

In this scenario we have a drastic expansion of the Universe during the early period of the Big Bang. In this case, the background solutions are:

$$
s=2 \quad, \quad r=\omega+\frac{1}{2} .
$$

Strictly speaking, inflation occurs for $\omega \geq \frac{1}{2}$.

\section{$2.3 \quad$ Radiation $\left(\alpha=\frac{1}{3}\right)$}

In this era the energy density of the Universe was tottaly dominated by relativistic particles. The solutions are:

$$
s=0 \quad, \quad r=\frac{1}{2} .
$$

As it is easily seen the above solutions are the same for the General Relativity by the same scenario 14 .

\subsection{Incoherent Matter $(\alpha=0)$}

This phase is such that the Universe is dominated by nonrelativistic matter with negligible pressure. The solutions are:

$$
s=2-3 r \quad, \quad r=\frac{2+2 \omega}{4+3 \omega} .
$$

\section{Perturbed Equations}

Our objective here is to calculate the perturbations of the equations (6), (7) and $(\mathbb{8})$, and to obtain the exact solutions for the perturbed equations for 
each scenario of the Universe. We are interested in the density matter perturbations so that we will only use the temporal components of the Einstein's equations since that component is directly associated with the scalar modes. Adopting the synchronous gauge, we put $h_{0 \mu}=0$.

In order to derive the perturbed equations, we write the metric tensor as:

$$
\widetilde{g}_{\mu \nu}=g_{\mu \nu}+h_{\mu \nu},
$$

leading to the Ricci tensor

$$
\widetilde{R}_{00}=R_{00}+\delta R_{00},
$$

where

$$
\delta R_{00}=\frac{1}{a^{2}}\left[\ddot{h}_{k k}-2 \frac{\dot{a}}{a} \dot{h}_{k k}+2\left(\frac{\dot{a}^{2}}{a^{2}}-\frac{\ddot{a}}{a}\right) h_{k k}\right] .
$$

The energy-momentum tensor is given by

$$
\widetilde{T}^{00}=T^{00}+\delta T^{00}=T^{00}+\delta \rho ;
$$

the energy-momentum tensor trace is

$$
\widetilde{T}=T+\delta T=T+\delta \rho-3 \delta p ;
$$

for the d'Alembertian of the scalar field we find

$$
\delta \square \phi=\delta \ddot{\phi}+a \dot{a} h^{k k} \dot{\phi}-\frac{1}{2 a^{2}} \dot{h}_{k k} \dot{\phi}+3 \frac{\dot{a}}{a} \delta \dot{\phi}-\frac{\nabla^{2}}{a^{2}} \delta \phi .
$$

Using now the following definitions

i) $h_{k k}=a^{2} h$,

ii) $\delta \phi=\lambda \phi \quad$ with $\lambda<<1$, 
iii) $\delta \rho=\Delta \rho$ with $\Delta<<1$,

we will get the set of perturbed equations:

$$
\begin{gathered}
\frac{1}{2} \ddot{h}+\frac{\dot{a}}{a} \dot{h}=\frac{8 \pi}{\phi} \rho(\Delta-\lambda)\left(\frac{2+\omega+3 \omega \alpha+3 \alpha}{3+2 \omega}\right)+\ddot{\lambda}+2 \dot{\lambda} \frac{\dot{\phi}}{\phi}(1+\omega), \\
\ddot{\lambda}+\dot{\lambda}\left(2 \frac{\dot{\phi}}{\phi}+3 \frac{\dot{a}}{a}\right)+\lambda\left(\frac{\ddot{\phi}}{\phi}+3 \frac{\dot{a}}{a} \frac{\dot{\phi}}{\phi}\right)-\frac{1}{2} \dot{h} \frac{\dot{\phi}}{\phi}-\frac{1}{a^{2}} \nabla^{2} \lambda= \\
=\frac{8 \pi}{(3+2 \omega)} \frac{\Delta}{\phi} \rho(1-3 \alpha), \\
\dot{\Delta}-(1+\alpha)\left(\frac{1}{2} \dot{h}-\delta U^{k}, k\right)=0, \\
(1+\alpha)(2-3 \alpha) \frac{\dot{a}}{a} \delta U^{j}+\delta \dot{U}^{j}(1+\alpha)=-a^{-2} \alpha \Delta_{, j} g^{j k} .
\end{gathered}
$$

Equations (25), (26) are the differential equation for the perturbation $h(t)$ of the gravitational field, $\lambda(t)$ is the scalar field perturbation and $\Delta(t)$, the perturbation for ordinary matter. Equations (27) and (28) are the perturbed equations derived from the conservation equation (\$). With the above set of differential second order equations, we will analyse each phase of the Universe.

In what follows, we will suppose that the perturbations have a plane wave behaviour:

$$
\lambda(x, t)=\lambda(t) \exp (-i \vec{q} \cdot \vec{x}) \text { and } \quad h(x, t)=h(t) \exp (-i \vec{q} \cdot \vec{x})
$$

where $q$ is the wavenumber of the perturbations.

\subsection{Vacuum $\quad(\rho=0)$}

In the Universe without matter there is only perturbations of the metric and scalar field. The two coupled linear second-order equation for $h(t)$ and $\lambda(t)$ 
are given by:

$$
\begin{gathered}
\frac{1}{2} \ddot{h}+\frac{r}{t} \dot{h}=\ddot{\lambda}+2 \frac{\dot{\lambda}}{t}(1+\omega)(1-3 r), \\
\frac{1}{2} \dot{h}(1-3 r)=\ddot{\lambda} t+2 \dot{\lambda}(1-3 r)+3 \dot{\lambda} r+\frac{1}{t^{2 r-1}} q^{2} \lambda .
\end{gathered}
$$

Differentiating (31) and removing $\dot{h}$ and $\ddot{h}$, we find the following linear third-order differential equation for $\lambda(t)$ :

$$
\begin{gathered}
\dddot{\lambda}+\ddot{\lambda}\left[\frac{2}{t}(1+r)\right]+ \\
+\dot{\lambda}\left[\frac{r}{t^{2}}(16+12 \omega)-\frac{r^{2}}{t^{2}}(24+18 \omega) \frac{1}{t^{2}}(2+2 \omega)+\frac{q^{2}}{t^{2 r}}\right]+ \\
+\lambda\left(\frac{q^{2}}{t^{2 r}}\right)=0 .
\end{gathered}
$$

In order to solve the above equation, we perform the following substitution:

$$
\begin{gathered}
\lambda=\frac{\kappa}{t}, \quad \dot{\kappa}=t^{r} \gamma, \quad x=t^{p}, \text { with } p=1-r, \\
\gamma=x^{m} g, \text { with } m=\frac{1-2 r}{1-r}, \quad y=\frac{x q}{1-r} .
\end{gathered}
$$

We get from equation (32),

$$
y^{2} g^{\prime \prime}+y g^{\prime}+g\left(y^{2}-1\right)=0,
$$

i.e., Bessel equation of order 1 , where the prime denotes derivatives with respect to the conformal time.

The solutions of (33) are the Bessel and Neumann functions of the first kind:

$$
g=C_{1} J_{1}(y)+C_{2} N_{1}(y) .
$$


The solutions for $\lambda(t)$ and $h(t)$ are given by:

$$
\begin{gathered}
\lambda(t)=\frac{1}{t}\left[\int t^{1-r}\left(C_{1} J_{1}(y)+C_{2} N_{1}(y)\right) d t+C_{3}\right] \\
h(t)=\int\left[\frac{1}{(1-3 r) t^{2}}\left[6 r+\frac{2 q^{2}}{t^{2 r-4}}\right]\left[\int t^{1-r}\left(C_{1} J_{1}(y)+C_{2} N_{1}(y)\right) d t+C_{3}\right]+\right. \\
+\frac{1}{(1-3 r) t^{r}}\left[4-6 r-\frac{4}{t}(1+r)\right]\left[C_{1} J_{1}(y)+C_{2} N_{1}(y)\right]+ \\
\left.+\frac{4}{(1-3 r) t^{r}}\left[C_{1} \dot{J}_{1}(y)+C_{2} \dot{N}_{1}(y)\right]\right] d t+C_{4}
\end{gathered}
$$

where $C_{1}, C_{2}, C_{3}$ and $C_{4}$ are constants and $\dot{J}_{k}, \dot{N}_{k}$ means respectively the time derivative of Bessel and Neumann functions.

\subsection{Inflation $(\alpha=-1)$}

The remarkable feature of this case is that the density contrast is null. This can be seen through equations (27)-(28). So, we have two coupled linear second-order equation for $h(t)$ and $\lambda(t)$ :

$$
\begin{gathered}
\ddot{h}+\frac{2 r}{t} \dot{h}-2 \ddot{\lambda}-\frac{4}{t}(2 r+1) \dot{\lambda}-\frac{16 \pi}{(1+r)} r \rho \lambda=0, \\
\ddot{\lambda}+2 \dot{\lambda}\left(\frac{2+3 r}{t}\right)+\frac{1}{t^{2 r+2}} q^{2} \lambda-\frac{1}{t} \dot{h}+\frac{16 \pi}{t^{2}(1+r)} \rho \lambda=0 .
\end{gathered}
$$

The resolution method is very similar to the vacuum case. So we find the following linear third-order differential equation for $\lambda(t)$ :

$$
\begin{gathered}
\dddot{\lambda}+\ddot{\lambda}\left(\frac{5 r+3}{t}\right)+\dot{\lambda}\left(\frac{1}{t^{2 r}} q^{2}+\frac{\left(6 r^{2}+6 r-2\right)}{t^{2}}\right)+ \\
+\lambda\left(\frac{1}{t^{2 r+1}} q^{2}+\frac{\left(6 r^{2}-4 r-2\right)}{t^{3}}\right)=0 .
\end{gathered}
$$


By employing the transformations:

$$
\begin{gathered}
\lambda=\frac{\kappa}{t}, \quad \dot{\kappa}=t^{r} \gamma, \quad x=t^{p} \quad \text { with } p=1-r, \\
\gamma=x^{m} g \quad \text { with } \quad m=\frac{1-7 r}{2(1-r)}, \quad y=\frac{x q}{1-r},
\end{gathered}
$$

we obtain the Bessel equation of the order $k$ :

$$
y^{2} g^{\prime \prime}+y g^{\prime}+\left(y^{2}-k^{2}\right) g=0
$$

where

$$
k=\frac{r+3}{2(1-r)}
$$

Its solution is

$$
g(y)=C_{1} J_{k}(y)+C_{2} J_{-k}(y),
$$

where $J_{k}(y)$ is the Bessel function of the order $k$.

The solutions for $\lambda(t)$ and $h(t)$ are given by:

$$
\begin{gathered}
\lambda(t)=\frac{1}{t}\left[\int t^{\frac{1-5 r}{2}}\left(C_{1} J_{k}(y)+C_{2} J_{-k}(y)\right) d t+C_{3}\right] \\
h(t)=\int\left[t ^ { - \frac { 1 + 5 r } { 2 } } \left[\left(\frac{1-5 r}{2}\right)\left(C_{1} J_{k}(y)+C_{2} J_{-k}(y)\right)+\right.\right. \\
+t^{-\frac{5 r}{2}}\left[\left(\frac{1-5 r}{2}\right)\left(C_{1} \dot{J}_{k}(y)+C_{2} \dot{J}_{-k}(y)\right)\right]+ \\
+\frac{(3 r+2)}{t}\left[-\frac{1}{t^{2}}\left(\int t^{\frac{1-5 r}{2}}\left(C_{1} J_{k}(y)+C_{2} J_{-k}(y)\right) d t+C_{3}\right)+\right. \\
\left.+\frac{1}{t}\left[\int t^{\frac{1-5 r}{2}}\left(C_{1} J_{k}(y)+C_{2} J_{-k}(y)\right)\right]\right]+ \\
\left.+\left(\frac{(6 r+2)}{t^{2}}+\frac{1}{t^{2 r}} q^{2}\right)\left[\int t^{\frac{1-5 r}{2}}\left(C_{1} J_{k}(y)+C_{2} J_{-k}(y)\right) d t+C_{3}\right]\right] d t+C_{4}
\end{gathered}
$$

where $C_{1}, C_{2}, C_{3}, C_{4}$ are constants. 
In contrast with the vacuum case, $r$ is greater than 1 for $\omega>\frac{1}{2}$. For $r=1\left(\omega=\frac{1}{2}\right)$, equation (39) becomes an Euler equation and admit the particular power-law solution:

$$
\lambda=t^{n}, \quad n=-2 \pm \sqrt{4-q^{2}} .
$$

\subsection{Radiation $\left(\alpha=\frac{1}{3}\right)$}

By considering the background solutions for the perturbed quantities, we have, from equations (25)-(28)

$$
\begin{gathered}
\ddot{h}+\frac{1}{t} \dot{h}=2 \ddot{\lambda}+\frac{3}{2 t^{2}}(\Delta-\lambda), \\
\ddot{\lambda}+\frac{3}{2 t} \dot{\lambda}+\frac{1}{t} q^{2} \lambda=0, \\
\dot{\Delta}=\frac{2}{3} \dot{h}-\frac{4}{3} \delta U^{j},{ }_{j}, \\
\delta U^{j}+2 t \delta \dot{U}^{j}=-\frac{1}{2} \Delta^{, j} .
\end{gathered}
$$

Taking equation (46) and performing the following transformations:

$x=t^{p} \quad$ with $p=\frac{1}{2}, \lambda=x^{m} g \quad$ with $m=-\frac{1}{2}$ and $\quad y=2 q x$,

we obtain the differential Bessel equation:

$$
g^{\prime \prime}+\frac{1}{y} g^{\prime}+\frac{1}{y^{2}}\left[y^{2}-\left(\frac{1}{2}\right)^{2}\right] g=0 \text {. }
$$

The solution for $\lambda(t)$ is:

$$
\lambda(t)=t^{-\frac{1}{4}}\left(C_{1} J_{\frac{1}{2}}(y)+C_{2} J_{-\frac{1}{2}}(y)\right),
$$

where $C_{1}$ and $C_{2}$ are constants. 
Now, from equations (45),(47) and the equation (48),we have two coupled linear differential second-order equations to solve:

$$
\begin{gathered}
\ddot{h}+\frac{1}{2 t} \dot{h}-\frac{3}{2} \ddot{\Delta}-\frac{3}{4 t} \dot{\Delta}-\frac{1}{2 t} q^{2} \Delta=0, \\
\ddot{h}+\frac{1}{t} \dot{h}=2 \ddot{\lambda}+\frac{3}{2 t^{2}}(\Delta-\lambda) .
\end{gathered}
$$

Subtracting these two equations we have:

$$
\dot{h}=-3 t \ddot{\Delta}-\frac{3}{2} \dot{\Delta}-\left(q^{2}-\frac{3}{t}\right) \Delta+F(\lambda)
$$

where

$$
F(\lambda)=4 t \ddot{\lambda}-\frac{3}{t} \lambda
$$

With the equation (53) and equations (51), we obtain the differential third-order non-homogeneous equation for $\Delta(t)$ :

$$
\dddot{\Delta}+\frac{5}{2 t} \ddot{\Delta}+\left(\frac{1}{3 t} q^{2}-\frac{1}{2 t^{2}}\right) \dot{\Delta}+\left(\frac{1}{2 t^{3}}+\frac{1}{3 t^{2}} q^{2}\right) \Delta=\mathcal{F}(\lambda)
$$

where

$$
\mathcal{F}(\lambda)=\frac{1}{3 t} \dot{F}(\lambda)+\frac{1}{6 t^{2}} F(\lambda)
$$

Performing the transformations:

$$
\Delta=\frac{\kappa}{t} \quad \text { and } \quad \dot{\kappa}=t \gamma
$$

we obtain the following differential second-order non-homogeneous equation:

$$
\ddot{\gamma}+\frac{3}{2 t} \dot{\gamma}+\frac{1}{3 t} q^{2} \gamma=\mathcal{F}(\lambda)
$$

whose solution for $\Delta(t)$ is: 


$$
\begin{gathered}
\Delta(t)=\frac{1}{t}\left[\int t ^ { \frac { 5 } { 4 } } \left[\bar{C}_{1} J_{\frac{1}{2}}(z)+\bar{C}_{2} J_{-\frac{1}{2}}(z)+\right.\right. \\
+\frac{q}{\sqrt{3}} J_{\frac{1}{2}}(z) \int \mathcal{F}(\lambda(t)) t^{-\frac{1}{2}}\left(\frac{J_{-\frac{1}{2}}(z)}{W\left(\frac{1}{2},-\frac{1}{2}\right)}\right) d t+ \\
\left.\left.+\frac{q}{\sqrt{3}} J_{-\frac{1}{2}}(z) \int \mathcal{F}(\lambda(t)) t^{-\frac{1}{2}}\left(\frac{J_{\frac{1}{2}}(z)}{W\left(\frac{1}{2},-\frac{1}{2}\right)}\right) d t\right] d t\right]+\frac{1}{t} C_{3},
\end{gathered}
$$

where $z=2 q t^{\frac{1}{2}} / \sqrt{3}$ and $W\left(\frac{1}{2},-\frac{1}{2}\right)=J_{\frac{1}{2}}^{\prime} J_{-\frac{1}{2}}-J_{\frac{1}{2}} J_{-\frac{1}{2}}^{\prime}$ the Wronskian constructed with the Bessel functions.

Equations (50) and (58) are the solutions for scalar field perturbation and for density perturbation.

\subsection{Incoherent Matter $(\alpha=0)$}

Here, we have the following set of coupled differential equations:

$$
\begin{gathered}
\ddot{h}+\frac{2 r}{t} \dot{h}=\frac{16 \pi}{t^{2}}(\Delta-\lambda)\left(\frac{2+\omega}{3+2 \omega}\right)+2 \ddot{\lambda}+4 \dot{\lambda} \frac{(2-3 r)}{t}(1+\omega), \\
\ddot{\lambda}+\dot{\lambda}\left(\frac{4}{t}-\frac{3 r}{t}\right)+\lambda\left(\frac{2}{t^{2}}-\frac{3 r}{t^{2}}\right)-\frac{1}{2} \dot{h} \frac{(2-3 r)}{t}+ \\
+\frac{q^{2}}{t^{2 r}} \lambda=\frac{8 \pi}{t^{2}}(3+2 \omega) \Delta, \\
\dot{\Delta}-\left(\frac{1}{2} \dot{h}-\delta U_{k}^{k}\right)=0, \\
\delta \dot{U}^{j}+\frac{2 r}{t} \delta U^{j}=0 .
\end{gathered}
$$

Setting the four-velocity perturbation $\delta U^{k}$ null, which, in this case, is allowed by one infinitesimal gauge transformation, we have:

$$
(\ddot{\Delta}-\ddot{\lambda})+\frac{2 r}{t}(\dot{\Delta}-\dot{\lambda})+\frac{(2 r-2)}{t^{2}}(\Delta-\lambda)=0
$$


whose solution is

$$
\Delta=\lambda+t^{2(1-r)}
$$

Substituing (64) in (60), we obtain:

$$
\ddot{\lambda}+\frac{2}{t} \dot{\lambda}+\frac{1}{t^{2 r}} q^{2} \lambda=\frac{6 r^{2}-13 r+6}{t^{2 r}} .
$$

Now, perfoming the transformations:

$x=t^{p} \quad$ with $p=1-r \quad, \quad \lambda=x^{m} g \quad$ with $m=-\frac{1}{2(1-r)} \quad, \quad y=\frac{x q}{1-r}$, we obtain the differential second-order non-homogeneous equation:

$$
y^{2} g^{\prime \prime}+y g^{\prime}+\left[y^{2}-\left(\frac{1}{2(1-r)}\right)^{2}\right] g=\frac{6 r^{2}-13 r+6}{(1-r)^{2}} .
$$

Its solution is given by:

$$
\begin{gathered}
g(y)=\frac{6 r^{2}-13 r+6}{(1-r)^{2}}\left[J_{\nu}(y) \int \frac{1}{y^{2}}\left(\frac{J_{-\nu}(y)}{W(\nu,-\nu)}\right) d y+\right. \\
\left.+J_{-\nu}(y) \int \frac{1}{y^{2}}\left(\frac{J_{\nu}(y)}{W(\nu,-\nu)}\right) d y\right]+\bar{C}_{1} J_{\nu}(y)+\bar{C}_{2} J_{-\nu}(y)
\end{gathered}
$$

where $\nu=-m$.

The solutions for $\lambda(t)$ and $\Delta(t)$ are:

$$
\begin{gathered}
\lambda(t)=\frac{6 r^{2}-13 r+6}{q t^{\frac{1}{2}}}\left[J_{\nu}(y) \int \frac{1}{t^{2-r}}\left(\frac{J_{-\nu}(y)}{W(\nu,-\nu)}\right) d t+\right. \\
\left.+J_{-\nu}(y) \int \frac{1}{t^{2-r}}\left(\frac{J_{\nu}(y)}{W(\nu,-\nu)}\right) d t\right]+ \\
+\left(\bar{C}_{1} J_{\nu}(y)+\bar{C}_{2} J_{-\nu}(y)\right) t^{-\frac{1}{2}}, \\
\Delta(t)=\frac{6 r^{2}-13 r+6}{q t^{\frac{1}{2}}}\left[J_{\nu}(y) \int \frac{1}{t^{2-r}}\left(\frac{J_{-\nu}(y)}{W(\nu,-\nu)}\right) d t+\right.
\end{gathered}
$$




$$
\begin{gathered}
\left.+J_{-\nu}(y) \int \frac{1}{t^{2-r}}\left(\frac{J_{\nu}(y)}{W(\nu,-\nu)}\right) d t\right]+ \\
+\left(t^{-\frac{1}{2}}\right)\left(\bar{C}_{1} J_{\nu}(y)+\bar{C}_{2} J_{-\nu}(y)\right)+t^{2(1-r)} .
\end{gathered}
$$

The equations above are the solutions for $\lambda(t)$ and $\Delta(t)$ for $r \neq 1$.

On the other hand for $r=1$ equation (63)- 65) admits the particular solution $\Delta=\lambda+C_{3} t^{-1}+C t e$, where

$$
\lambda=t^{m}, \quad m=\frac{-1 \pm \sqrt{1-4 q^{2}}}{2} .
$$

\section{Asymptotic Behaviour}

In this section we analyse the solutions obtained above by calculating their asymptotic expressions 15. We can readly see that the arguments of the Bessel functions found in the last section are indeed the ratio beetwen the physical wavelength $\lambda_{f}=a / q$ of the perturbation and the particle-horizon distance $H^{-1}$. We have, then, $\lambda_{f}>H^{-1}$ when $t \rightarrow 0$ and $\lambda_{f}<H^{-1}$ when $t \rightarrow \infty$. These relations invert when $r>1$.

In what follows, for sake of simplicity, we have eliminated all gauge modes appearing in the solutions [16], which are represented by the constant $C_{3}$, and we have chosen null phases for the oscillating terms.

\section{$4.1 \quad$ Vacuum}

i) $t \rightarrow 0$

$$
\begin{gathered}
\lambda(t) \simeq C_{1} t^{2(1-r)}+C_{2} \\
h(t) \simeq \frac{C_{1}}{1-3 r}\left(2 t^{1-2 r}+q^{2} t^{5-4 r}-2 r t^{-2 r}\right)+
\end{gathered}
$$




$$
+\frac{C_{2}}{1-3 r}\left(q^{2} t^{3-2 r}+2 t^{-1}+(4+2 r) t^{-2}\right)
$$

ii) $t \rightarrow \infty$

$$
\begin{aligned}
& \lambda(t) \simeq t^{\frac{r-1}{2}}\left[A_{1} \cos \left(t^{1-r}\right)+A_{2} \sin \left(t^{1-r}\right)\right], \\
& h(t) \simeq t^{\frac{r-1}{2}}\left[A_{1} \sin \left(t^{1-r}\right)+A_{2} \cos \left(t^{1-r}\right)\right],
\end{aligned}
$$

with $A_{1}$ and $A_{2}$ given by some functions of $C_{1}$ and $C_{2}$. We also recall that

$$
r=\frac{\omega+1 \mp \sqrt{1+\frac{2 \omega}{3}}}{4+3 \omega} .
$$

\subsection{Inflation}

i) for $t \rightarrow 0$

$$
\begin{gathered}
\lambda(t) \simeq C_{1} t^{2(1-r)}+C_{2} t^{-(1+3 r)} \\
h(t) \simeq \sum_{i=1}^{6} C_{i} t^{z_{i}}[\cos (t)]+\sum_{i=1}^{6} D_{i} t^{z_{i}}[\sin (t)] ;
\end{gathered}
$$

ii) for $t \rightarrow \infty$

$$
\begin{gathered}
\lambda(t) \simeq t^{-2(1+r)}\left[C_{1} \cos \left(t^{1-r}\right)+C_{2} \sin \left(t^{1-r}\right)\right], \\
h(t) \simeq \sum_{i=1}^{7} A_{i} t^{x_{i}}+\sum_{i=1}^{7} B_{i} t^{y_{i}},
\end{gathered}
$$

where $A_{i}, B_{i}, C_{i}$ and $D_{i}$ are constants and the exponents are given by:

$$
\begin{array}{lll}
x_{1}=2-3 r, & x_{2}=\frac{7}{2}-5 r, & x_{3}=\frac{3}{2}-3 r, \quad x_{4}=3-\frac{3}{2} r, \\
x_{5}=5-\frac{7}{2} r, & x_{6}=\frac{7}{2}-\frac{7}{2} r, & x_{7}=\frac{11}{2}-\frac{11}{2} r \quad ; \\
y_{1}=-1-4 r, & y_{2}=-1-\frac{13}{2} r, & y_{3}=-3-\frac{9}{2} r, \quad y_{4}=-3-\frac{11}{2} r, \\
y_{5}=1-\frac{11}{2} r, & y_{6}=-\frac{5}{2}-\frac{11}{2} r, & y_{7}=-\frac{1}{2}-\frac{15}{2} r ; \\
z_{1}=-\frac{5}{4}-2 r, & z_{2}=-\frac{1}{2}-3 r, & z_{3}=-\frac{5}{2}-2 r \\
z_{4}=-\frac{1}{2}-2 r, & z_{5}=-2-2 r, & z_{6}=-4 r .
\end{array}
$$


The constants above are all combinations of $C_{1}$ and $C_{2}$. Finally we recall that

$$
r=\omega+\frac{1}{2}
$$

\subsection{Radiation}

i) $t \rightarrow 0$

$$
\begin{aligned}
& \lambda(t) \simeq C_{1}+C_{2} t^{\frac{1}{2}} \\
& \Delta(t) \simeq C_{1} t+C_{2} t^{\frac{1}{2}}
\end{aligned}
$$

ii) $t \rightarrow \infty$

$$
\begin{gathered}
\lambda(t) \simeq t^{-\frac{1}{2}}\left[C_{1} \sin \left(t^{\frac{1}{2}}\right)+C_{2} \cos \left(t^{\frac{1}{2}}\right)\right], \\
\Delta_{h}(t) \simeq C_{1}^{\prime} \sin \left(t^{\frac{1}{2}}\right)+C_{2}^{\prime} \cos \left(t^{\frac{1}{2}}\right) .
\end{gathered}
$$

The non-homogeneous part of the equation (55) gives, by a direct inspection, a mode that in the asymptotic regime decays with time.

\subsection{Incoherent Matter}

i) $t \rightarrow 0$

$$
\begin{gathered}
\lambda(t) \simeq C_{1}+C_{2} t^{-1}+A(r, q) t^{r-2}, \\
\Delta(t) \simeq \lambda(t)+t^{2(1-r)} ;
\end{gathered}
$$

ii) $t \rightarrow \infty$

$$
\begin{gathered}
\lambda(t) \simeq t^{\frac{-2+r}{2}}\left[C_{1} \sin \left(t^{1-r}\right)+C_{2} \cos \left(t^{1-r}\right)\right]+A t^{\frac{6 r-5}{2}} \sin \left(t^{1-r}\right), \\
\Delta(t) \simeq \lambda(t)+t^{2(1-r)},
\end{gathered}
$$


we recall that

$$
r=\frac{2+2 \omega}{4+3 \omega} .
$$

\section{Analyses of the Results}

\subsection{Vacuum Case}

In the vacuum case we verify that the exponent of the background solutions is always less than unity so that, for $t \rightarrow 0$, both $\lambda(t)$ and $h(t)$ have growing modes. On the other hand for $t \rightarrow \infty, \lambda(t)$ and $h(t)$ have only oscillating decaying modes. It means that perturbation of the scalar field and the perturbation of gravitational field whose wavelengths are greater than particlehorizon are growing perturbation in contrast with those having wavelengths smaller than the particle-horizon which are all decaying perturbations, as it is an expected result. This result generalizes those obtained in [17].

\subsection{Inflation Case}

We have in the present phase three cases: $r<1, r=1$ and $r>1$. (The case $r=1$ represents the transition between two regime in the same phase: inflationary expansion and "soft expansion".)

a) $r=1$ : this can represents the transition between two regime along the same phase: inflationary expansion and "soft expansion". From equation (44) we have,

i) for $q<2, \lambda(t)$ has decayind modes,

ii) for $q>2, \lambda(t)$ has oscillating modes. 
b) $r<1$ : there is no inflationary inflation, in spite of the fact that $\alpha=-1$. Moreover, when $\omega<-1 / 2$, we will find an important growth of $\lambda(t)$, but a decaying scale factor. For $t \rightarrow 0, \lambda(t)$ has one growing mode and one decaying mode, while $h(t)$ has only decaying oscillating mode. For $t \rightarrow \infty$, $\lambda(t)$ has only decaying oscillating mode and $h(t)$ show growing modes.

c) $r>1$ : this is the case where there is strictly inflation. The scalar filed grows at power $t^{2}$ and the scale factor undergoes huge expansion for $\omega>1 / 2$. For $t \rightarrow 0$ all modes of $\lambda(t)$ are oscillating decaying and $h(t)$ has gentle grow modes for $1<r<2(1 / 2<\omega<3 / 2)$. For $t \rightarrow \infty, \lambda(t)$ and $h(t)$ are completly decreasing perturbations.

\subsection{Radiation Case}

In this case, $r=1 / 2$ and the background evolves as if the scalar field were constant. For $t \rightarrow 0, \lambda(t)$ and $\Delta(t)$ (density perturbation) undergoes gentle

growing, as $t$ and $t^{\frac{1}{2}}$. For $t \rightarrow \infty$ both perturbations show only decaying oscillating modes. In this radiation case the scalar perturbation gives a decaying contribuition to the corresponding GR results.

\subsection{Incoherent Matter Case}

Here, we have also three case $r=1, r<1$, and $r>1$. The behaviour of $\Delta(t)$ is the same of $\lambda(t)$ plus one term in form of power-law.

a) $r=1$ : from particular solution from equation (65) we see that i) for $q<1 / 2, \lambda(t)$ has decaying modes; 
ii) for $q>1 / 2, \lambda(t)$ has two oscillating modes with decaying amplitude.

$\Delta(t)$ has a supplementary constant term.

b) $r>1$ : in this case for $t \rightarrow 0, \lambda(t)$ has oscillating modes, one of then with growing amplitude. The behaviour of $\Delta(t)$ shows also a pure decreasing mode. For $t \rightarrow \infty, \lambda(t)$ and $\Delta(t)$ have only decaying modes, except for the case $r>2(-3 / 2<\omega-4 / 3)$. We note that in this case we have also a superluminal expansion of the background.

c) $r<1$ : only $\Delta(t)$ has a growing mode for $t \rightarrow 0$. $\lambda(t)$ has only decaying oscillating modes and $\Delta(t)$ is still increasing, for $t \rightarrow \infty$. In the case of $-4 / 3<\omega<-1$ we have $r<0$ and then $\Delta(t)$ is growing with high power of $t$. We observe that, for this case, we have a contracting Universe. So it can be consider of physical interest if it can be applied to some primordial phase.

Our results confirm those obtain in (20), where it is pointed out that the BD solution of this case can not leads to a solution of the structure formation problem, when we consider values of $\omega$ imposed by local physics.

\section{Conclusion}

We gave in this paper a general classification of perturbation in BD Theory corresponding to the phases of the expanding universe where the matter is described by equations of state $p=\alpha \rho$ with $\alpha=0, \alpha=-1, \alpha=1 / 3$ and $\rho=0$. We assumed that the background solution for the scalar field and for the scale factor are that of "pure" power-law. We have found the 
exact solutions of the perturbed equations in the form of integrals of Bessel functions. The calculation here confirm, in general, the very known fact: perturbations at scales larger than particle horizon distance grow up, while for smaller scales the perturbation exhibits an oscillating behaviour. It is not true for incoherent matter where density perturbations can be always amplificated.

In the inflationary phase we can see that the strong expansion of the universe erases the perturbation on the scalar field but the gravitational perturbation is growing for scales greater than particle-horizon distance. On the other hand, it is very important to emphasize that the perturbations in the density of the matter are null in Brans-Dicke inflation. This recovers the main results from classical inflationary scenario.

The radiation phase do not give an expressive contribution with respect to the GR corresponding results. In the incoherent matter the situation is more rich. For some negative values of $\omega$ we can have a superluminal expansion and at the same time a significant amplification of perturbations.

\section{Acknowledgements}

We have benefited from many enlightening discussions with Prof. M. Novello and we also thank D.W.L. Monteiro for comments. S.V.B. Gonçalves thanks CAPES (Brazil) for financial support. This work was partially supported by $C N P q($ Brazil). 


\section{References}

[1] C. Brans and R. H. Dicke, Phys. Rev. 124, 925(1961).

[2] P.A.M. Dirac, Proc. Roy. Soc. (London) A165, 199(1938).

[3] P. Jordan, Z. Physik 157, 112(1959).

[4] G.C. McVittie, M.N.R.A. Soc. 183, 749(1978).

[5] A.H. Guth, Phys. Rev. D 23, 347(1981).

[6] D. La e P.J. Steinhardt, Phys. Rev. Let. 62, 376(1989).

[7] P.J. Steinhardt and F. S. Accetta, Phys. Rev. Lett. 64, 2740(1990).

[8] J.P. Baptista, A.B. Batista e J.C. Fabris, Rev. Bras. de Física 14, 20(1984).

[9] M. Kuwahara, H. Suzuki and E. Takasugi, Phys. Rev. D 15, 661(1994).

[10] T. Hirai and K. Maeda, Astrophys. J. 431, 6(1994).

[11] E.M. Lifschitz and I.M. Khalatnikov, ,Usp. Fiz. Nauk. 80, 391(1963);

E.M. Lifschitz, Zh. E. T. F.16, 587(1946);

[12] J.M. Bardeen, Phys. Rev. D22, 1882(1980).

[13] S.J. Kolitch and D.M. Eardley, Ann. of Phys. 241, 128(1995).

[14] S. Weinberg, Gravitation and Cosmology, Wiley, New York(1972). 
[15] M.A. Abramowitz e I. A. Stegun, Handbook of Mathematical Functions, Natl. Bur. Stand. Appl. Math. Ser. $n^{o} 55$.

[16] P.J.E. Peebles, The Large Scale Structure of the Universe, Princeton University Press, New Jersey(1980).

[17] J.C. Fabris and J. Martin, Phys. Letters B316, 476(1993). 